\title{
Kinetics of laser-induced low-temperature crystallization of amorphous silicon
}

\author{
Yu. L. Khait, R. Beserman, A. Chack, and R. Weil \\ Solid State Institute and Department of Physics, Technion-Israel Institute of Technology, Haifa 32000, Israel \\ W. Beyer \\ Institut für Photovoltaik Forschungszentrum, Jülich 52425, Germany
}

(Received 6 May 2002; accepted 30 August 2002)

\begin{abstract}
A brief report on experimental and theoretical studies of the kinetics of the laser-induced crystallization (LIC) in undoped amorphous hydrogenated silicon is presented. It is shown that the LIC occurs at a substantially lower temperature and occurs at this temperature much faster compared to the thermal crystallization in a furnace. A nanoscopic kinetic electron-related model of the LIC is presented. The model explains the experimental observations as the integral effect of a huge amount of nanoscale picosecond atomic and electronic reconstructions leading to more stable material states which are generated by electron-assisted short-lived (picosecond) large energy fluctuations in nanometer material regions. (C) 2002 American Institute of Physics.
\end{abstract}

[DOI: $10.1063 / 1.1516875$ ]

The laser-induced crystallization (LIC) of hydrogenated silicon $(a-\mathrm{Si}: \mathrm{H})$ has a considerable potential for technological applications to high-performance thin-film transistors and other devices. ${ }^{1-3}$ That is why the understanding of the LIC mechanism and ways to control the LIC of $a-\mathrm{Si}: \mathrm{H}$ are of significant practical importance. However, the LIC kinetic mechanisms are still not clear in spite of extensive studies and impressive achievements in the field. In this letter, we present a brief report on the results of recent experimental studies of the LIC in low-temperature undoped $a$-Si:H films and on a kinetic electron-related LIC model. This model predicts the main expected observations and suggests an explanation of experimental results, as we will see next. The proposed model is an extension to LIC of our nanoscopic kinetic models successfully applied to a broad range of processes taking place in various amorphous and crystalline material; ${ }^{4-14}$ many of these results have been summarized in Refs. 6 and 7 and reviewed in Ref. 8. These applications include continuous wave laser-induced structural changes in $a$-Si:H (and other amorphous materials), ${ }^{4,6}$ the thermal crystallization in $a-\mathrm{Si}: \mathrm{H}^{5,6}$ and amorphous silicon $(a-\mathrm{Si}),{ }^{9}$ as well as the thermal recrystallization and intermixing in $\mathrm{Si} / \mathrm{Ge}$ superlattices of nanometer periods disordered by ion implantation. ${ }^{10}$ The aforementioned structural changes in metastable amorphous materials include the following phenomena which can also be extended to the LIC kinetics in $a$-Si:H. First, the observed crystallization includes a huge number of diffusionlike hoppings of $\mathrm{Si}$ atoms toward more ordered states related to a lower free energy. In the course of the crystallization, each of the $\mathrm{Si}$ atoms involved experiences on the average $\nu_{A}=5-10$ hoppings.

Second, the hoppings of $\mathrm{Si}$ atoms are generated by nanoscale short-lived (picosecond) large energy fluctuations (SLEFs) of atomic particles up to their peak thermal energy (per atom) $\varepsilon_{i p} \geqslant \Delta E \gg k T$ ( $T$ is temperature and $k$ is the Boltzmann constant). The SLEF-generated hyperthermal fluctuating atoms are able to overcome the energy barriers $\Delta E$ $\gg k T$ and perform diffusionlike jumps into more ordered po- sitions related to a lower free energy. ${ }^{4-6,9,11,12}$ Third, SLEFs and SLEF-induced atomic diffusion jumps in $a$-Si are seen in computer simulations. ${ }^{12}$ SLEF-induced local picosecond material distortions generate local downward electron transitions which accompany the atomic hoppings reduce the activation energy $\Delta E$ and enhance exponentially their probability. ${ }^{4-6,9,7,13,10}$ This reduces exponentially crystallization time ${ }^{4-7,13}$

$$
t_{N}=\Delta \tau \nu_{A} \exp \left(\Delta G / k T_{A}\right)
$$

found from the kinetic consideration. Here $\Delta \tau$ $=10^{-13}-10^{-12} \mathrm{~s}$ is the SLEF lifetime which determines the duration of SLEF-induced atomic hoppings. The parameter $\Delta G=\Delta E-T \Delta S$ is the activation free energy. $\Delta S$ is the related local entropy change. The value $\Delta E=E-\delta n\langle\Delta e\rangle$ is the SLEF threshold energy sufficient for a fluctuating atom to overcome the diffusion energy barrier with the "assistance" of $\delta n$ downward electron transitions. $\langle\Delta e\rangle$ is the average interlevel distance related to the electron transitions. ${ }^{4-7,9,11,13}$ Here, $E$ is the local energy barrier not affected by electron transitions (i.e., when $\delta n \rightarrow 0$ ).

From Eq. (1) one can find the crystallization temperature

$$
T_{A}=\frac{\Delta E}{k}\left[\ln \left(\frac{t_{N}}{\Delta \tau \cdot \nu_{A}}\right)+\frac{\Delta S}{k}\right]^{-1},
$$

which is expected during the LIC time $t_{N}$. The temperature $T_{A}$ can be measured through the Raman Stokes-anti-Stokes ratio. The laser generates in $a$-Si:H high nonequilibrium concentrations of electrons and holes and breaks down a great number of interatomic $\mathrm{Si}-\mathrm{Si}$ bonds. This promotes an enhancement of the number $\delta n$ of downward electron transitions (during atomic reconstructions) and causes a reduction of $E, \Delta E, \Delta G$, and $T_{A}$ [Eqs. (1) and (2)]. Rough estimates discussed below give $\Delta E \approx 1.5 \mathrm{ev}, \Delta G \approx 1.75 \mathrm{eV}$ and $T_{A}$ $\approx 600 \mathrm{~K}$ (for $t_{N} \approx 10 \mathrm{~min}$ ). This temperature is substantially 


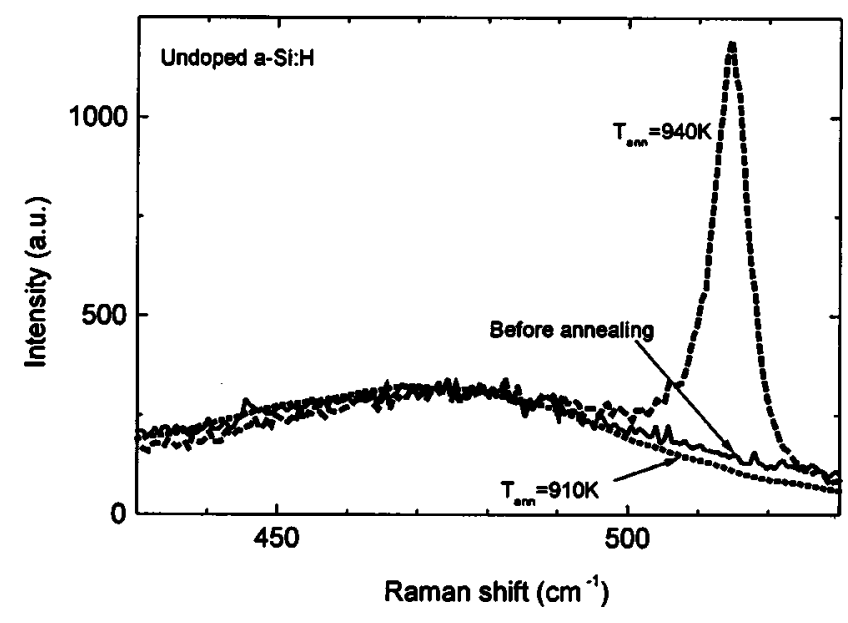

FIG. 1. Raman spectra of undoped $a$-Si:H films before and after the thermal crystallization in vacuum (of $10^{-6}$ Torr) for $30 \mathrm{~min}$. Unannealed: continuous line; annealed at $910 \mathrm{~K}$ : dotted line; annealed at $940 \mathrm{~K}$ : dashed line.

lower than the temperature $\approx 950 \mathrm{~K}$ obtained for the thermal crystallization in a furnace. The experimental observations confirm this conclusion (Figs. 1 and 2).

In our experimental studies, we compare the onset of crystallization by two methods, laser and furnace annealing. The samples were undoped $a-\mathrm{Si}: \mathrm{H}$ films of $1 \mu \mathrm{m}$ thickness, grown on quartz substrates at $500{ }^{\circ} \mathrm{C}$ by plasma enhanced chemical vapor deposition from undiluted $\mathrm{SiH}_{4}$. Backscattering Raman spectra were used to determine the onset of crystallization and its time dependence, and to measure the local temperature during laser annealing. The spectra were taken at room temperature, using the $514.5 \mathrm{~nm}$ wavelength of an Ar laser focused on a spot diameter of $\approx 0.5 \mu \mathrm{m}$. The laser was operated at a power of $\approx 10-15 \mathrm{~mW}$ for laser annealing. For the furnace annealed samples, a power of less than $5 \mathrm{~mW}$ was used for monitoring the onset of crystallization; at this power, no laser crystallization was observed even after several hours exposure. The crystalline structure is characterized by a Raman peak around $520 \mathrm{~cm}^{-1}$, the exact frequency and width of which depend on the size of microcrystals. ${ }^{15,16}$ The onset of crystallization, was detected by the appearance of the $\sim 500 \mathrm{~cm}^{-1}$ Raman peak. For the temperature measurement during laser annealing, we used the corrected Stokes to anti-Stokes ratio which gives us a

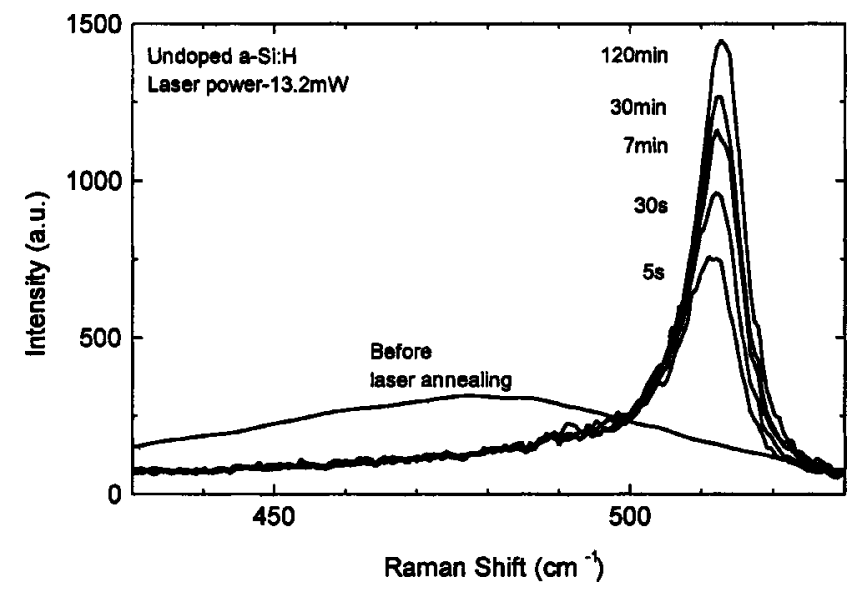

FIG. 2. Raman spectra of undoped $a$-Si:H films before and after laser-

induced crystallization for various times and fixed laser power. Where $\sigma_{T}^{2}=\overline{(\Delta r)^{2}}$. The carrier concentrations $n_{e}(r, z)$ and
Downloaded 21 Dec 2006 to 134.94.122.39. Redistribution subject to AlP license or copyright, see http://apl.aip.org/apl/copyright.jsp measurement of the local sample temperature with an accuracy better than $\pm 10 \%$ for both $\mathrm{cw}$ or pulsed laser illumination. ${ }^{15-17}$ A conventional tube furnace was used to anneal the samples under vacuum.

The furnace annealed samples remained uncrystallized when annealed at $910 \mathrm{~K}$ for half an hour, but crystallized when the annealing temperature was raised to $940 \mathrm{~K}$ for the same period of time. This is consistent with our previous crystallization experiments. ${ }^{18}$ The Raman spectra of these samples are shown in Fig. 1. The local temperature during laser annealing was measured to be $T_{A}=620 \mathrm{~K}$. Figure 2 shows the Raman spectra of the LIC samples exposed for different periods of time to a laser power of $13.2 \mathrm{~mW}$. Thus, the following experimental results have been obtained. First, the LIC takes place at temperatures $T_{A}$ substantially lower than furnace temperatures $T_{f}$ needed for the thermal crystallization. Second, the LIC onset in the laser-affected region of radius $R \approx 0.5 \mu$ requires only a few seconds, whereas the thermal crystallization in $a-\mathrm{Si}: \mathrm{H}$ at $T_{f}$ requires a substantially longer time. Many other samples were tested and yielded similar results.

Consider now an explanation of the experimental observations. cw laser-induced crystallization in $a$-Si:H takes place in nonequilibrium conditions characterized by the following phenomena and parameters. First, high nonequilibrium concentrations $n_{e}$ and $n_{h}$ of electrons and holes (i.e., electron-hole plasma) are generated in the laser affected material volume $V_{L} \approx \pi R_{L}^{2} \ell$ of radius $R_{L}$, and laser beam penetration depth $\ell$. Second, in the volume $V_{L}$ there exists a high nonequilibrium concentration $n_{B}$ (of the order of $n_{e}$ ) of broken interatomic bonds which are produced in the course of photon-induced electron excitations. Third, the laser beam produces heating of the material atoms in $V_{L}$ which can be characterized by the effective temperature $T_{A}$ measured by the Raman Stokes-anti-Stokes ratio.

As we mentioned earlier, our nanoscopic kinetic SLEFbased model takes into account the two kinds of laserinduced athermal phenomena. The first is the high concentration $n_{B}$ of broken $\mathrm{Si}-\mathrm{Si}$ interatomc bonds that reduces the energy $E$ [in Eqs. (1) and (2)] by about $30 \%$ of the value of $E \approx 3 \mathrm{eV}$ in the furnace annealing. ${ }^{18}$ Thus $E \approx 2 \mathrm{eV}$ for the LIC. The second, $\delta n \approx n_{e} v_{e} \Sigma_{t} \Delta \tau \approx 5$ downward electron transitions ${ }^{4-7,9,13}$ accompanying every SLEF-induced atomic hopping reduce additionally $\Delta G$ and $\Delta E$ [in Eqs. (1) and (2)], as well as $T_{A}$ to the aforementioned values $\Delta G \approx 1.75 \mathrm{eV}, \Delta E \approx 1.5 \mathrm{eV}$, and $T_{A} \approx 600 \mathrm{~K}$ in agreement with the experimental observations. Here, $\langle\Delta e\rangle \approx 0.1 \mathrm{eV},{ }^{5} \Sigma_{t}$ is the cross section of downward electron transitions, $v_{e}$ is the electron velocity. These results present the parameters averaged over a laser spot.

Local parameters $n_{e}(r, z), \quad n_{h}(r, z), \quad n_{B}(r, z), \quad$ and $T_{A}(r, z)$ depend on the distance $r$ from the laser beam axis (or from the spot center) and the distance $z \leqslant \ell$ from the material surface within the volume $V_{L}$. For instance, in many cases, one can assume that $T_{A}(r, z)$ is a Gaussian function of $r$ (at given $z$ )

$$
T_{A}(r, z)=T_{A}(r=0, z) \times \exp \left[-r^{2} / 2 \sigma_{T}^{2}(z)\right],
$$


$n_{h}(r, z)$, as well as the concentration $n_{B}(r, z)$ of broken interatomic bonds, also decrease when $r$ increases.

Then one finds [from Eqs. (1) and (3)] an extremely sharp dependence of the crystallization time on $r$

$$
t_{N}(r, z)=\nu_{A} \Delta \tau \times \exp \left[\frac{\Delta G(r, z)}{k T_{A}(r=0, z)} \cdot \exp \left(\frac{r^{2}}{2 \sigma_{T}^{2}(z)}\right)\right],
$$

which is much stronger than the exponential one, especially when $r>\sigma_{T}$.

From Eq. (3), one can conclude that in regions close to the laser spot center local temperatures can be higher and the crystallization time $t_{N}$ shorter. The experiment confirms this expectation and shows that the first onset of the LIC appears after the time $t_{N_{1}} \approx 5 \mathrm{~s}$ (Fig. 2).

Using Eq. (1) one can estimate the local temperature $T_{L}$ in regions of a smaller distance $r<0.5 \mu$ which produces the crystallization onset after $t_{N} \approx 5 \mathrm{~s}$ (Fig. 2). Assuming the same $\Delta G \approx 1.75 \mathrm{eV}$, one finds $T_{L} \approx 700 \mathrm{~K}>T_{A} \approx 600 \mathrm{~K}$ higher than the aforementioned Stokes-anti-Stokes temperature $T_{A}$ averaged over the region of radius $R \approx 0.5 \mu$, but lower than $T_{f} \approx 940 \mathrm{~K}$ found for the furnace crystallization.

In summary, the main results reported are:

(i) LIC takes place much faster and at substantially lower temperatures compared to the furnace crystallization; and

(ii) the observed LIC is the integral effect of a huge amount of nanoscale picosecond thermal-athermal atomic and electronic interrelated reconstructions toward more ordered material states.
This research was supported in part by grants from the Bundesministerium fur Bildung, Forschung, and Technology (BMBF), Germany through the Israel Ministry of Science and the Israel Ministry of Absorption.

${ }^{1}$ M. Ivanda, K. Furic, O. Gamulin, M. Persin, and D. Gracin, J. Appl. Phys. 70, 4637 (1991).

${ }^{2}$ R. Z. Bachrach, K. Winer, J. B. Boyce, S. E. Ready, R. I. Johnson, and G. B. Anderson, Electron. Mater. 19, 241 (1990).

${ }^{3}$ L. P. Avakyants, V. S. Gorelik, I. A. Kurova, and A. V. Chervyakov, Solid State Phys. 39, 1925 (1997).

${ }^{4}$ I. Abdulhalim, R. Beserman, Y. L. Khait, and R. Weil, Appl. Phys. Lett. 55, 1170 (1987).

${ }^{5}$ Y. L. Khait and R. Weil, J. Appl. Phys. 78, 6504 (1995).

${ }^{6}$ Y. L. Khait, Monograph, Kinetics and Applications of Atomic Diffusion in Solids: Nanoscopic Electron-Affected Stochastic Dynamics (Scitech, Switzerland, 1997).

${ }^{7}$ Y. L. Khait, Phys. Rep. 99, 237 (1983).

${ }^{8}$ K. R. Rao, Curr. Sci. 75, 1328 (1998).

${ }^{9}$ Y. L. Khait and R. Beserman, Phys. Rev. B 33, 2983 (1986).

${ }^{10}$ W. Freiman, R. Beserman, Y. L. Khait, M. Shaanan, K. Dettmer, and R. Kessler, Phys. Rev. B 48, 2282 (1993); Selected Papers on Quantum Well Intermixing for Photonics, edited by E. H. Li and B. Thompson (SPEI Pub. 1998).

${ }^{11}$ Y. L. Khait, R. Beserman, W. Beyer, R. Weil, and H. Wagner, Phys. Rev. B 42, 9000 (1990).

${ }^{12}$ Y. L. Khait, A. Silverman, R. Weil, and J. Adler, Phys. Rev. B 44, 8308 (1991).

${ }^{13}$ Y. L. Khait, J. Salzman, and R. Beserman, Appl. Phys. Lett. 53, 2135 (1988).

${ }^{14}$ Y. L. Khait, V. Garber, E. Bahir, and I. Snapiro, Appl. Phys. Lett. 79, 2990 (2001).

${ }^{15}$ Z. Iqbal, A. R. Weeb, and S. Veprek, Appl. Phys. Lett. 36, 163 (1980).

${ }^{16}$ H. W. Lo and A. Compaan, J. Appl. Phys. 51, 1565 (1980).

${ }^{17}$ A. Compaan, M. C. Lee, and G. J. Trott, Phys. Rev. B 32, 6731 (1985).

${ }^{18}$ F. Edelman, C. Cytermann, R. Brener, M. Eizenberg, Y. L. Khait, R. Weil, and W. Beyer, J. Appl. Phys. 75, 7875 (1994). 